\title{
Relationship between duration of type 2 diabetes mellitus and stress levels based on age, gender, anthropometry and vital signs
}

\author{
Dinesh Thangavel $^{1 *}$, Vijaiananth Pitchaipillai ${ }^{2}$, Venkatesan Rangan ${ }^{1}$, \\ Anbarasi Muthusamy ${ }^{1}$, Raghuvaran Sivaprakasam ${ }^{1}$
}

\author{
${ }^{1}$ Department of Physiology, Dhanalakshmi Srinivasan Medical College \& Hospital, Siruvachur, Perambalur-621113, \\ Tamil Nadu, India \\ ${ }^{2}$ Department of General Medicine, Dhanalakshmi Srinivasan Medical College \& Hospital, Siruvachur, Perambalur- \\ 621113, Tamil Nadu, India
}

Received: 03 October 2015

Accepted: 20 October 2015

*Correspondence:

Dr. Dinesh Thangavel,

E-mail: sclerombbs@yahoo.co.in

Copyright: (C) the author(s), publisher and licensee Medip Academy. This is an open-access article distributed under the terms of the Creative Commons Attribution Non-Commercial License, which permits unrestricted non-commercial use, distribution, and reproduction in any medium, provided the original work is properly cited.

\begin{abstract}
Background: The incidence of diabetes mellitus is increasing at a rapid rate and by 2030 the case load would be doubled. Stress and various other factors such as physical and psychological factors play a significant role in the worsening of hyperglycemia in patients with type 2 diabetes mellitus. Aim and objective was to study the relationship between duration of type 2 diabetes mellitus and stress levels based on age, gender, anthropometry and vital signs.

Methods: After obtaining approval from the institute ethics committee for human studies, the present study was conducted in the Department of Physiology, Dhanalakshmi Srinivasan Medical College and Hospital, Perambalur, Tamil Nadu on 50 type 2 diabetes mellitus patients ( 25 males, 25 females). After obtaining informed, written consent the following parameters such as duration of diabetes mellitus (Months), anthropometric parameters such as Body Mass Index (BMI), Waist Hip Ratio (WHR), Perceived Stress Scale (PSS) Scores, Vital signs viz. Pulse Rate (PR), Systolic Blood Pressure (SBP), Diastolic Blood Pressure (DBP) and Respiratory Rate (RR) were recorded in all the study participants. Data for all the parameters was collected as per the study protocol and computerized in Microsoft excel database. All statistical analyses were done at $5 \%$ level of significance and $\mathrm{P}<0.05$ was considered as statistically significant.

Results: Average age of male and female volunteers was $52.16 \pm 12.20$ years and $55.68 \pm 9.68$ years respectively. Statistical analysis using independent Student's ' $t$ ' test, showed that there was no significant gender difference on the above mentioned parameters. Pearson's correlation showed a statistically significant association between duration of diabetes mellitus and BMI $(\mathrm{P}<0.01)$. Correlation between duration of diabetes mellitus and PSS scores was also statistically significant $(\mathrm{P}=0.02)$.

Conclusions: Results of the present study indicate that stress and increase in BMI are important independent risk factors for the worsening of hyperglycemia. When there is presence of other co morbid conditions the risk may be increased or it may affect the management or prognosis.
\end{abstract}

Keywords: Type 2 diabetes mellitus, Perceived stress, Gender, Anthropometry, Vital signs

\section{INTRODUCTION}

The incidence of diabetes mellitus is increasing rapidly and by 2030 the case load would be doubled. Also estimates state that every fifth person with diabetes would be from India. ${ }^{1}$ Type 2 diabetes mellitus is a metabolic disorder that is characterized by hyperglycemia in context of insulin resistance and relative insulin deficiency. ${ }^{2}$ 
Stress and various other factors such as physical and psychological factors play a significant role in the development of hyperglycemia in the setting of type 2 diabetes mellitus. Also it has been shown to have major effects on metabolic activity by stimulating the release of various hormones that result in elevated blood glucose levels. ${ }^{4}$ This is of adaptive importance in a healthy organism, whereas in diabetes stress-induced increase in glucose could not be metabolized properly, owing to an impaired insulin levels. ${ }^{3}$

The Perceived Stress Scale (PSS) Scores was developed by Sheldon Cohen and his colleagues in 1983. It has become one of the most widely used psychological instruments for measuring nonspecific perceived stress. ${ }^{7}$ It has been used in studies assessing the effectiveness of stress-reducing interventions and the extent to which there are associations between psychological stress and psychiatric and physical disorders. ${ }^{6}$ The present study was planned to study the relationship between the duration of type 2 diabetes and the stress levels based on age, gender, anthropometry and vital signs.

\section{METHODS}

The present study was conducted in the Department of Physiology, Dhanalakshmi Srinivasan Medical College and Hospital, Perambalur, Tamil Nadu on 50 Type 2 diabetes mellitus patients (25 males, 25 females) attending the General Medicine OPD.

The purpose of the study, procedures and benefits were explained to them in detail. The willing participants who have been diagnosed as type 2 diabetic patients were recruited for the study by getting informed, written consent. Subjects receiving medication for any other chronic ailment, known cardiac or hypertensive patients, smokers and alcoholics were excluded from the study.

\section{Parameters}

The parameters assessed includes age (years), gender (Male, Female), duration of diabetes mellitus (Months), Anthropometric parameters such as Body Mass Index (BMI), Waist Hip Ratio (WHR), vital signs viz. Pulse Rate (PR), Systolic Blood Pressure (SBP), Diastolic Blood Pressure (DBP) were recorded after 10 minutes of rest in supine position using digital BP monitor (CitizenCH 432B, Japan) and Respiratory Rate (RR) was recorded passively by observing the abdominal movements. Then the participants were interviewed for assessing stress levels using Perceived Stress Scale (PSS) Questionnaire -10- item Analysis on a one to one basis. Each item is rated on a 5-point scale ranging from never (0) to almost always (4) (Annexure-I).

Ethics: The study was conducted after obtaining clearance from the Institute ethics committee for human studies and caries less than minimal risks.

\section{Statistical analysis}

Data for all parameters were collected as per the study protocol and computerized in Microsoft excel database. Variables in both genders were compared by using Student's paired t-test.

\section{RESULTS}

Average age of male $(n=25)$ and female $(n=25)$ volunteers was $52.16 \pm 12.20$ years and $55.68 \pm 9.68$ years respectively. Statistical analysis using independent Student's 't' test, showed that there was no significant difference between male and female patients on the above mentioned parameters. The details are given in Table 1 . Pearson's correlation showed a statistically significant association between duration of diabetes mellitus and BMI $(\mathrm{P}<0.01)$. Correlation between duration of diabetes mellitus and PSS Scores was also statistically significant $(\mathrm{P}=0.02)$.

Table 1: Baseline characteristics of the study participants: Age, gender, BMI, WHR, PR, SBP, DBP, RR, duration of diabetes mellitus and PSS.

\begin{tabular}{|lll|}
\hline Parameters & $\begin{array}{l}\text { Male }(\mathbf{n}=25) \\
(\text { Mean } \pm \text { SD })\end{array}$ & $\begin{array}{l}\text { Female }(\mathbf{n}=25) \\
(\text { Mean } \pm \text { SD })\end{array}$ \\
\hline Age $($ years $)$ & $52.16 \pm 12.20$ & $55.68 \pm 9.68$ \\
\hline BMI $\left(\mathrm{kg} / \mathrm{m}^{2}\right)$ & $26.29 \pm 3.74$ & $25 \pm 2.8$ \\
\hline WHR & $1.15 \pm 0.05$ & $1.1 \pm 0.1$ \\
\hline PR $(\mathrm{b} / \mathrm{m})$ & $80.08 \pm 5.52$ & $83.6 \pm 3.1$ \\
\hline SBP $(\mathrm{mmHg})$ & $129.2 \pm 18.9$ & $138 \pm 12.91$ \\
\hline DBP $(\mathrm{mmHg})$ & $82 \pm 15$ & $87.6 \pm 8.79$ \\
\hline RR $(\mathrm{b} / \mathrm{m})$ & $18.2 \pm 2.31$ & $19.6 \pm 1.21$ \\
\hline Duration $(\mathrm{months})$ & $31.76 \pm 26.89$ & $40.32 \pm 30.67$ \\
\hline PS score & $23.72 \pm 3.04$ & $22.68 \pm 3.68$ \\
\hline
\end{tabular}

Table 2: Shows the relationship between age, duration of diabetes mellitus (months), BMI, WHR, PR, SBP, DBP, RR with PSS of the study participants $(n=50)$.

\begin{tabular}{|lll|}
\hline Paired sample & $\begin{array}{l}\text { Pearson's } \\
\text { correlation } \\
\text { coefficient }(r)\end{array}$ & $\begin{array}{l}\text { Two tailed } \\
\text { 'p' value }\end{array}$ \\
\hline Age and PSS & +0.163 & 0.29 \\
\hline Duration and PSS & $+0.621^{* *}$ & $<0.01$ \\
\hline BMI and PSS & $+0.317^{*}$ & 0.02 \\
\hline WHR and PSS & +0.92 & 0.53 \\
\hline SBP and PSS & +0.226 & 0.11 \\
\hline DBP and PSS & +0.224 & 0.12 \\
\hline
\end{tabular}

$* \mathrm{P}<0.05, * * \mathrm{P}<0.01$

There was a trend towards increase in PSS Scores with vital signs parameters such as PR, SBP, DBP and RR, which were not statistically significant. The details are given in Table 1 \& Table 2. 


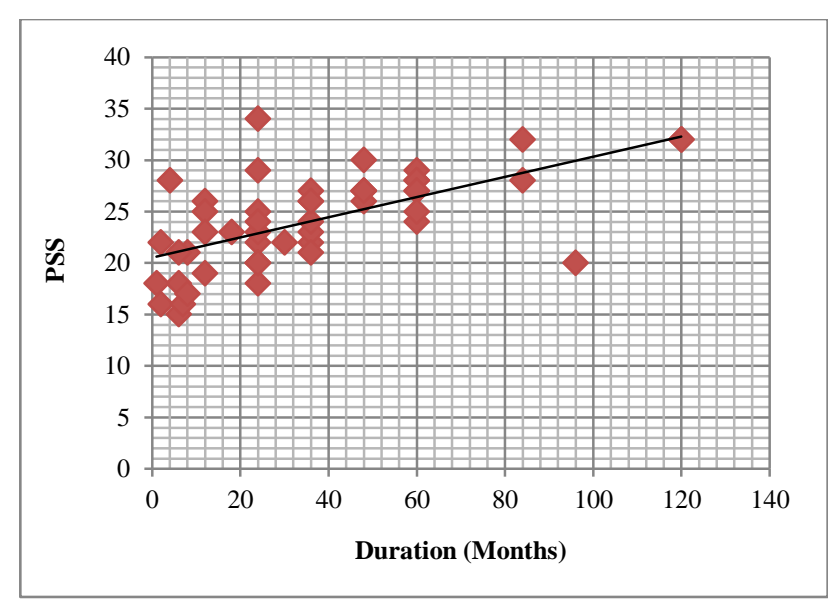

Figure 1: Relationship between of duration of diabetes mellitus (months) and PSS $(n=50)$.

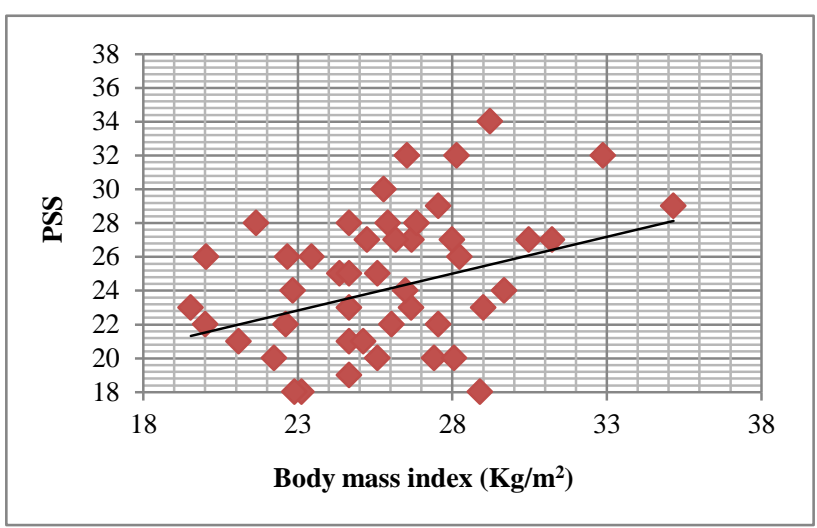

Figure 2: Shows the relationship between BMI and PSS.

\section{DISCUSSION}

Type 2 diabetes is typically a metabolic disorder resulting in a shorter life span with a ten-year-shorter life expectancy. ${ }^{2}$ Various lifestyle factors are known to be important in the development of type 2 diabetes, including obesity (defined by a body mass index of greater than thirty), junk food and lack of physical activity, stress and urbanization. ${ }^{3}$

Stress refers to the perceived or actual threat on physical and/or psychological homeostasis of the human body. Disrupted homeostasis elicits the so called "stress response", meaning the activation of central and peripheral neuroendocrine mechanisms responsible for various adaptive responses behaviors. Stress may play a role in the onset of diabetes; it can have a detrimental effect on glycemic control and can affect lifestyle. ${ }^{5}$ In our study, a significant positive correlation had been observed between duration of diabetes and PSS scores which clearly implies that stress is a major influencing factor which in turn can worsen the diabetes adversely especially when the person is of longer diabetic age and obese. In both male and female patients, the average PSS scores were more than 20 which mean that the stress levels were severe.

The clinical implications of this research illustrate the need for a better understanding of the effects of stress, as well as a serious acceptance of the need for psychosocial support for people in this predicament. The impact of stress on diabetes is clearly varied and may depend on other psychosocial factors. One of these is social support, and research has shown this may provide a buffering effect in times of stress. ${ }^{4,9,10}$

Results of our study indicate that increase in BMI had positive association with the stress scores, which is in concordance with previous studies as the increase in body fat is generally associated with an increase in risk of metabolic disorders such as type 2 diabetes mellitus. ${ }^{11}$ Previous studies like The Study to Help Improve Early evaluation and management of risk factors Leading to Diabetes (SHIELD) 2004 screening questionnaire (mailed survey) and the National Health and Nutrition Examination Surveys (NHANES) 1999-2002 (interview, clinical and laboratory data) were conducted on the nationally representative samples $\geq 18$ years old. Previous studies like, The Study to Help Improve Early evaluation and management of risk factors Leading to Diabetes (SHIELD, 2004) screening questionnaire (mailed survey) and the National Health and Nutrition Examination Surveys (NHANES, 1999-2002)were conducted on the nationally representative samples $\geq 18$ years old. Responses were received from 127420 of 200000 households (64\%, representing 211097 adults) for SHIELD, and 4257 participants for NHANES. In their study they reported an increase in the prevalence of metabolic disorders such as diabetes mellitus, hypertension and dyslipidemia. ${ }^{8,12}$ Also study by Abdulla et al. in 2010 mentioned that the overall relative risk of diabetes was higher for obese individuals compared to those with normal weight. ${ }^{13}$

Also end-stage complications of diabetes have the greatest perceived burden on quality of life. Comprehensive diabetes treatment and prevention of complications play a major role in ensuring a good quality of life. Stress relaxation techniques and good glycemic control also have favorable effects on the quality-of-life. ${ }^{14}$ PSS Scores around 13 are considered as average stress. Scores of 20 or higher are considered as high stress in which case intervention using stress reduction techniques have to be considered. ${ }^{7}$ Excessive psychological stress is associated with increased blood pressure, higher BMI, larger waist to hip ratio, shorter telomere length, higher cortisol levels, suppressed immune function, decreased sleep and increased alcohol consumption. In our previous pilot study which was done on 10 type 2 diabetic patients we had reported that stress significantly affects the management and compliance. ${ }^{4}$

In our study there was a statistically insignificant but definite trend towards increase in vital signs parameters 
such as PR, SBP, DBP and RR with PSS Scores. These changes might have been due to sympatho vagal imbalance which leads to hypermetabolic state, stressful state of mind and poor cardiac vagal tone. When the mind is in disturbed state, sympathetic activity increases and $\mathrm{RR}$ increases. Increase in sympathetic activity increases $\mathrm{PR}$ and increase in sympathetic tone in skeletal muscle, blood vessels, increases peripheral vascular resistance and hence, increase in SBP and DBP. ${ }^{15,16}$

\section{CONCLUSION}

We conclude that the results of the present study indicate that stress and increase in BMI are important independent risk factors for the development of hyperglycemia. When there is presence of other comorbid conditions the risk is increased or it may affect the management or prognosis. A more detailed study involving a larger population of diabetes patients is warranted for a concrete conclusion.

\section{Clinical implication}

Weight reduction by increasing the physical activities, stress reduction by Yoga, counseling sessions, relaxation techniques are to be considered as a routine treatment to the patients with diabetes. The authors wish to extend the study further with hundreds of participants and as well as a multi centric study.

\section{ACKNOWLEDGEMENTS}

Author would like to thank staff of the Physiology and General Medicine Departments and the Management of Dhanalakshmi Srinivasan Medical College and Hospital, Perambalur, Tamil Nadu who helped us to carry out this project.

\section{$\underline{\text { Annexure-I }}$}

\section{Cohen perceived stress scale questionnaire}

The following questions ask about your feelings and thoughts during The Past Month. In each question, you will be asked How Often you felt or thought a certain way. Although some of questions are similar, there are small differences between them and you should treat each one as a separate question. The best approach is to answer fairly quickly. That is, don't try to count up the exact number of times you felt a particular way, but tell me the answer that in general seems the best.

For each statement, please tell me if you have had these thoughts or feelings: never, almost never, sometimes, fairly often, or very often. (Read all answer choices each time)

\section{Never-0, Almost never-1, Sometimes-2, Fairly often-3, Very often-4}

B.1. In the past month, how often have you been upset because of something that happened unexpectedly? ..0, $1,2,3,4$

B.2. In the past month, how often have you felt unable to control the important things in your life?..........................0, $1,2,3,4$

B.3. In the past month, how often have you felt nervous or stressed?....................0,1, 2, 3, 4

B.4. In the past month, how often have you felt confident about your ability to handle personal problems?..................................................0, 1, 2, 3, 4

B.5. In the past month, how often have you felt that things were going your way? $0,1,2,3,4$

B.6. In the past month, how often have you found that you could not cope with all the things you had to do?. $. .0,1,2,3,4$

B.7. In the past month, how often have you been able to control irritations in your life? $0,1,2,3,4$

B.8. In the past month, how often have you felt that you were on top of things? $0,1,2,3,4$

B.9. In the past month, how often have you been angry because of things that happened that were outside of your control? $0,1,2,3,4$

B.10. In the past month, how often have you felt that difficulties were piling up so high that you could not overcome them?......................0, 1, 2, 3, 4 . 


\section{Perceived Stress (PSS) Score}

Each item is rated on a 5-point scale ranging from never (0) to almost always (4). Positively worded items are reverse scored, and the ratings are summed, with higher scores indicating more perceived stress. PSS-10 scores are obtained by reversing the scores on the four positive items:

For example, $0=4,1=3,2=2$, etc. and then summing across all 10 items. Items $4,5,7$, and 8 are the positively stated items.

\section{Your Perceived Stress Level was}

(Scores around 13 are considered average. Scores of 20 or higher are considered high stress.)

\section{Funding: No funding sources}

Conflict of interest: None declared

Ethical approval: The study was approved by the institutional ethics committee for human studies

\section{REFERENCES}

1. Wild S, Roglic G, Green A, Sicree R, King H. Global prevalence of diabetes: estimates for the year 2000 and projections for 2030. Diabetes Care. 2004;27(5):1047-53.

2. WHO. Diabetes Fact sheet $N^{\circ} 312$, October 2013. Available at: http://www.who.int/mediacentre/factsheets/fs312/en /. Accessed January 2015.

3. Dan L. Longo, Antony S. Fauci, Dennis L. Kasper, Stephen L. Hauser, J. Larry Jameson, Joseph Loscalzo. Diabetes mellitus. In: Dan L. Longo, Antony S. Fauci, Dennis L. Kasper, Stephen L. Hauser, J. Larry Jameson, Joseph Loscalzo, eds. Harrison's Principles of Internal Medicine. 18th ed. New York, NY: McGraw-Hill Professional; 2012: 2968-3002.

4. Dinesh Thangavel, Vijaiananth Pitchaipillai. Association of duration of illness and compliance with stress levels in type 2 diabetes mellitus patients - a pilot study. Natl J Physiol Pharmacol. 2014;4(3):249-51.

5. Kumar Vinay, Fausto Nelson, Abbas Abul K, Cotran Ramzi S, Robbins Stanley L. Stress. In: Kumar Vinay, Fausto Nelson, Abbas Abul K, Cotran Ramzi S, Robbins Stanley L, eds. Robbins and Cotran Pathologic Basis of Disease. 7th ed. Philadelphia: Saunders. 2005: 1194-1195.

6. Leon KA, Hyre AD, Ompad D, DeSalvo KB, Muntner P. Perceived stress among a workforce 6 months following hurricane Katrina. Soc Psychiatry Psychiatr Epidemiol. 2007;42(12):1005-11.

7. Cohen S, Kamarck T, Mermelstein R. A global measure of perceived stress. J Health Soc Behav 1983:24(4):385-96.

8. Cathy Lloyd, Julie Smith, Katie Weinger. Stress and diabetes: a review of the links. Diabetes Spectr. 2005;18(2):121-7.
9. Shlomo Melmed, Kenneth S. Polonsky, P. Reed Larsen, Henry M. Kronenberg. Stress and diabetes. In: Shlomo Melmed, Kenneth S. Polonsky, P. Reed Larsen, Henry M. Kronenberg, eds. Williams's Textbook of Endocrinology. 12th ed. Philadelphia: Elsevier/Saunders; 2011: 1371-1435.

10. Ganong WF. Cardiovascular regulatory mechanisms. In: Ganong WF, eds. Review of Medical Physiology. 22nd ed. San Francisco: McGraw Hill; 2005: 597-602.

11. Viner R, McGrath M, Trudinger P. Family stress and metabolic control in diabetes. Arch Dis Child. 1996;74:418-21.

12. Bays HE, Chapman RH, Grandy S. The relationship of body mass index to diabetes mellitus, hypertension and dyslipidaemia: comparison of data from two national surveys. Int $\mathbf{J}$ Clin Pract. 2007;61(5):737-47.

13. Abdullah A, Peeters A, de Courten M, Stoelwinder J. The magnitude of association between overweight and obesity and the risk of diabetes: a meta-analysis of prospective cohort studies. Diabetes Res Clin Pract. 2010;89(3):309-19.

14. Huang ES, Brown SE, Ewigman BG, Foley EC, Meltzer DO. Patient perceptions of quality of life with diabetes-related complications and treatments. Diabetes Care. 2007;30(10):2478-83.

15. Jerath R, Edry JW, Branes VA, Jerath V. Physiology of long pranayama breathing: Neural respiratory elements may provide a mechanism that explains how slow deep breathing shifts the autonomic nervous system. Med Hypotheses. 2006;67:56-71.

16. Dinesh T, Venkatesan R, Venkidusamy S. Effect of 12 weeks of pranayama training on basal physiological parameters in young, healthy volunteers. Panacea J Med Sci. 2014;4(1):28-30.

Cite this article as: Thangavel $\mathrm{D}$, Pitchaipillai V, Rangan V, Muthusamy A, Sivaprakasam R. Relationship between duration of type 2 diabetes mellitus and stress levels based on age, gender, anthropometry and vital signs. Int J Adv Med 2015;2:401-5. 